\title{
Nonlinear sliding friction of adsorbed overlayers on disordered substrates
}

\author{
Enzo Granato \\ Laboratório Associado de Sensores e Materiais, Instituto Nacional de Pesquisas Espaciais, 12245-970 São José dos Campos, \\ São Paulo, Brazil \\ S. C. Ying \\ Department of Physics, Brown University, Providence, Rhode Island 02912, USA \\ (Received 24 October 2003; revised manuscript received 22 December 2003; published 8 March 2004)
}

\begin{abstract}
We study the response of an adsorbed monolayer on a disordered substrate under a driving force using Brownian molecular-dynamics simulation. We find that the sharp longitudinal and transverse depinning transitions with hysteresis still persist in the presence of weak disorder. However, the transitions are smeared out in the strong disorder limit. The theoretical results here provide a natural explanation for the recent data for the depinning transition of $\mathrm{Kr}$ films on gold substrate.
\end{abstract}

DOI: $10.1103 /$ PhysRevB.69.125403

PACS number(s): 68.35.Gy, 68.35.Rh, 81.40.Pq

\section{INTRODUCTION}

Adsorbed overlayers under a driving force have attracted much interest recently ${ }^{1-11}$ as important examples of driven lattice systems. ${ }^{12,13}$ The nonlinear response of the overlayer in the presence of pinning centers resulted in various steady states, corresponding to a variety of different nonequilibrium dynamic phases. The nature of these dynamic phases and the transitions from one phase to the other are the microscopic factors controlling the macroscopic sliding friction and boundary lubrication. ${ }^{1,14-17}$ Other closely related systems include vortex lattices in type-II superconductors, ${ }^{18-23}$ chargedensity waves, ${ }^{24}$ and colloidal crystal layers. ${ }^{25}$ In general, both a periodic potential and disorder due to quenched defects are present in theses systems. In particular, in the sliding friction and boundary lubrication of surfaces the underlying crystal lattice provides the periodic pinning potential which competes with defect pinning centers due to impurities.

For periodic substrates, it has been shown through molecular dynamics simulation studies that there are different dynamical phases for the driven overlayer such as pinned solid, sliding solid, and liquid phases. The transitions between these phases often involve strong hysteresis effects, ${ }^{3}$ leading to important macroscopic behavior as the stick and slip motion, which can be observed experimentally with the surface force apparatus. ${ }^{14,15}$ Most of the theoretical results were based on two-dimensional models of overlayers with Lennard-Jones potential interaction between the adatoms. ${ }^{3}$ The results on these dynamical phases have been found to be universal and are reproduced even in models with pure elastic interacting potential. ${ }^{6}$ More recently, ${ }^{7}$ it has also been shown that, in the sliding state, the response of the overlayer to an additional force in the transverse direction can also lead to a depinning transition at low temperatures. Different sliding states also occur in presence of quasiperiodic substrates and have been investigated in detail recently for onedimensional models. ${ }^{9,10}$ However, the effects of disorder on the main features observed for two-dimensional systems, which can arise, for example, from point defects on a initially periodic substrate, have not been investigated satisfac- torily so far. Considerable analytical and numerical results are only available for driven lattice systems described by overdamped dynamics. ${ }^{12,13}$ Unfortunately, it is known that inertial effects significantly modify the nature of the depinning transition. ${ }^{1}$ Hence the results for these overdamped systems cannot be directly applied to the sliding friction problem involving adsorbed overlayers on a substrate.

In this work, we study numerically the nonlinear sliding friction of adsorbed overlayers on disordered substrates with a specific model of point defects, where the strength of the disorder can be varied by changing the concentration of the defects. We investigate the longitudinal and transverse response for weak and strong disorder by Brownian moleculardynamics simulations. We find that the main features, such as hysteresis, longitudinal, and transverse depinning transitions previously observed for periodic surfaces, ${ }^{3,7}$ still survive in the presence of weak disorder but are smeared out in the strong disorder limit. Recently, the depinning transition of $\mathrm{Kr}$ films on gold has been studied using the quartz crystal microbalance technique. ${ }^{17}$ Our results for the longitudinal response bear a strong qualitative resemblance to the experimental data of the depinning transition. In particular, it provides concrete theoretical support to the speculation that the aging effect on the depinning transition is due to the increasing disorder of the substrate.

\section{MODEL AND SIMULATION}

The model of interacting adatoms on a substrate with point disorder subject to an external driving force for the present study is a simple extension of the one previously used for periodic substrates. ${ }^{1,3,7}$ The dynamics is described by the Langevin equation

$$
m \ddot{\mathbf{r}_{i}}+m \eta \dot{\mathbf{r}}_{i}=-\frac{\partial U}{\partial \mathbf{r}_{i}}-\frac{\partial V}{\partial \mathbf{r}_{i}}+\mathbf{f}_{i}+\mathbf{F}
$$

where $\mathbf{r}_{\mathbf{i}}=\left(\mathbf{x}_{\mathbf{i}}, \mathbf{y}_{\mathbf{i}}\right)$ is the adsorbate position, $U=\Sigma_{i} u\left(r_{i}\right)$ is the substrate pinning potential, $V=\sum_{i \neq j} v\left(\mid \mathbf{r}_{\mathbf{i}}-\mathbf{r}_{\mathbf{j}}\right)$ is the interactions between adatoms, $\mathbf{F}$ is the uniform external force acting on each adatom, and $\mathbf{f}_{i}$ is a stochastic force, with zero 
average, and variance related to the microscopic friction parameter $\eta$, the mass of the particles $m$ and the temperature $T$ by the fluctuation dissipation relation

$$
\left\langle f_{i}^{\alpha}(t) f_{i}^{\alpha}\left(t^{\prime}\right)\right\rangle=2 \eta m k T \delta\left(t-t^{\prime}\right),
$$

where $\alpha$ represents Cartesian components. We choose a substrate potential which provides a periodic pinning for the overlayer except near a pinning center, given by the form

$$
u(\mathbf{r})=U_{0}[2-\cos (2 \pi x / a)-\cos (2 \pi y / a)] .
$$

Near a pinning center (point defect) located at $R_{p}$ $=\left(X_{p}, Y_{p}\right)$, the substrate potential is locally modified to the form

$$
u(\mathbf{r})=U_{p}[2-\cos (2 \pi x / a)-\cos (2 \pi y / a)]
$$

for

$$
\begin{aligned}
& X_{p}-a / 2<x<X_{p}+a / 2, \\
& Y_{p}-a / 2<y<Y_{p}+a / 2 .
\end{aligned}
$$

The strength of the random pinning potential $U_{p}$ is chosen to be uniformly distributed in a range of $4 U_{0}$ with an average $\left\langle U_{p}\right\rangle=U_{o}$. The position of the pinning centers $R_{p}$ $=\left(X_{p}, Y_{p}\right)$ is randomly chosen on a square lattice of lattice parameter $a$ and linear size $L$. With this choice, a pinning center consists of a square region with a force that vanishes smoothly at the boundary. For $U_{p}=U_{o}$, a constant, the entire substrate potential reduces to the periodic cosine potential in Eq. (3) studied previously. ${ }^{1,3,7}$ The strength of disorder is controlled by varying the concentration $x_{d}$ of randomly chosen pinning centers. In the dilute limit of $x_{d} \ll 1$, corresponding to weak disorder, this pinning potential has a predominant Fourier component at the wave vector $\vec{k}$ $=(2 m \pi / a, 2 n \pi / a)$. In the other limit of dense pinning centers with $x_{d} \rightarrow 1$, it represents an amorphous substrate with strong disorder. The interaction between adatoms is represented by a Lennard-Jones pair potential

$$
v\left(r_{i j}\right)=\epsilon\left[\left(r_{o} / r_{i j}\right)^{12}-2\left(r_{o} / r_{i j}\right)^{6}\right],
$$

where $\epsilon$ is the well depth and $r_{0}$ is the particle separation at the minima in the pair potential. We have chosen the parameters in the interaction potential to be $r_{o} / a \sim 1.5, \epsilon=U_{0}$, and the adsorbate coverage at $\theta=1 / 2$. This choice is made such that the ground state of the overlayer in the periodic substrate $\left(x_{d}=0\right)$ corresponds to the commensurate pinned $c(2 \times 2)$ structure studied previously. For the periodic substrate, the longitudinal and transverse response of the commensurate overlayer to an applied force in the present model have been extensively studied in connection with the sliding friction and boundary lubrication ${ }^{1,3,7}$ problem. Thus, this model provides a convenient starting point for the present study of disorder effects. In the discussions below we use dimensionless units normalizing the force by $2 \pi U_{o}$, velocities by $2 \pi U_{o} / \eta$ and temperature by $U_{0} / k_{B}$. We study the nonlinear response of the overlayer at different temperatures and pinning center concentrations via Brownian moleculardynamics simulations. ${ }^{26}$ Systems consisting of $N=50-800$
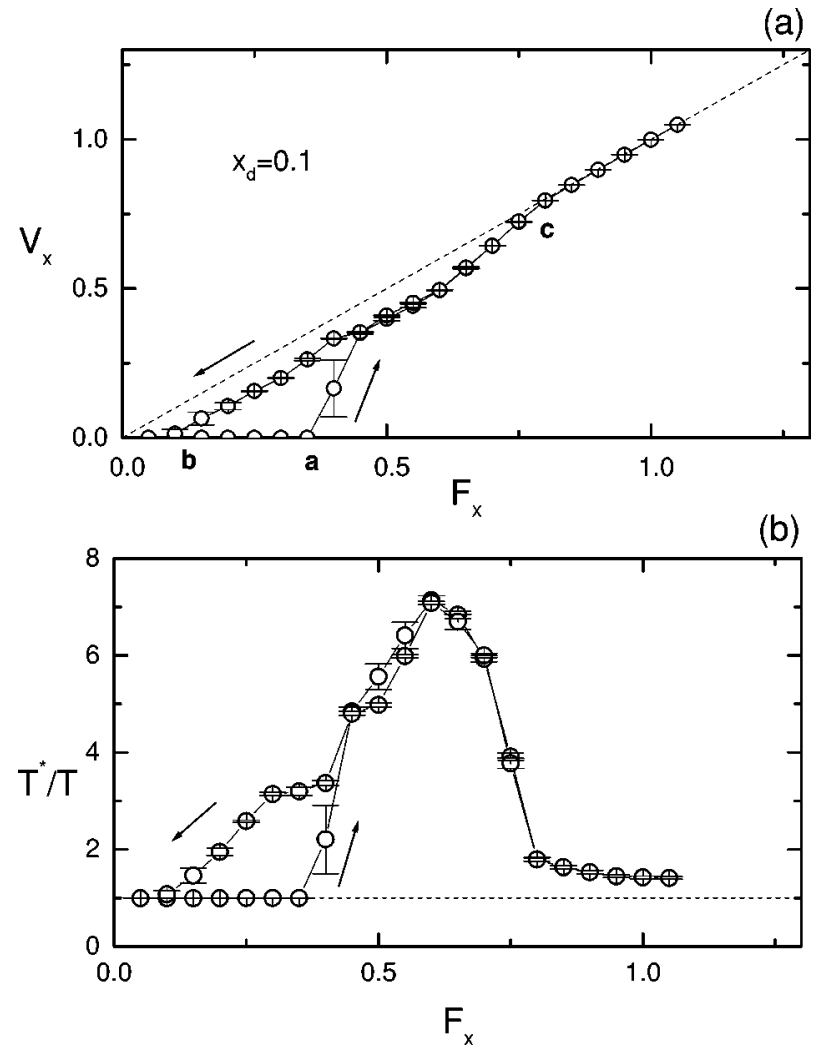

FIG. 1. Drift velocity $V_{x}$ (a) and effective temperature $T^{*}$ (b) of the overlayer as a function of the external force $F_{x}$, for dilute disorder $x_{d}=0.1$. The results are for $T=0.2$ and system size $L=20$. The direction of variation of $F_{x}$ is indicated by arrows.

adatoms were considered with the time variable discretized in units of $\delta t=0.002-0.01 \tau$, where $\tau=\left(m a^{2} / U_{o}\right)^{1 / 2}$. The main results were obtained for $\epsilon=U_{o}$ and $\eta=1$. For each set of parameters, calculations were performed for typically 1 $-4 \times 10^{5}$ time steps to allow the system to reach steady state. Then an equal number of time steps are used to evaluate time-averaged values of various physical quantities in this steady state. Finally, the results are also averaged over a number of (typically 6-11) different realizations of the disorder through the distribution of the $U_{p}$ value for the pinning centers.

\section{RESULTS AND DISCUSSION}

\section{A. Depinning transition and longitudinal response}

The longitudinal response to an applied force at a temperature $T=0.2$, well below the melting transition temperature of the $c(2 \times 2)$ phase on a periodic substrate, $T_{m}=1.2$, is shown in Figs. 1 and 2 for dilute $x_{d}=0.1$ and dense disorder $x_{d}=1$, respectively. In the former case, the main features are similar to that found for the periodic substrate..$^{3,7}$ The drift velocity $V_{x}$ is essentially zero below a critical value of $F_{a}$ (neglecting the tiny contribution from thermal creep motion which is always present for nonzero force). When the driving force exceeds the critical value $F_{a}$, there is a sharp depinning transition where the overlayer starts to slide and the drift velocity increases rapidly. Eventually a sliding re- 
(a)

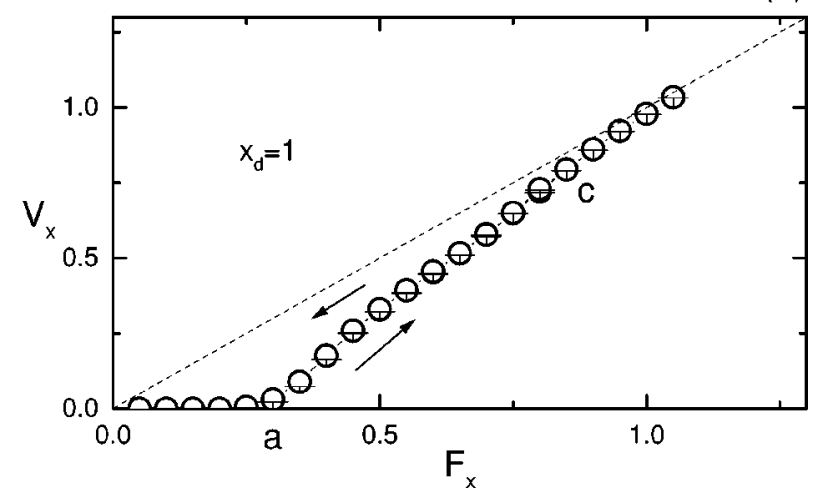

(b)

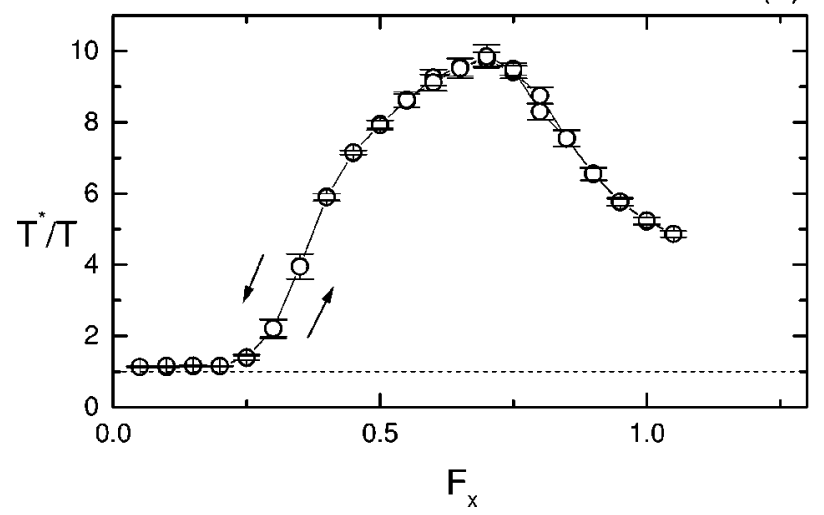

FIG. 2. Drift velocity $V_{x}$ (a) and effective temperature $T^{*}$ (b) of the overlayer as a function of the external force $F_{x}$, for dense disorder $x_{d}=1$. The results are for $T=0.2$ and system size $L$ $=20$. The direction of variation of $F_{x}$ is indicated by arrows.

gime for $F_{x} \gg F_{c}$ is reached where the longitudinal sliding friction, defined as $\bar{\eta}=F_{x} / V_{x}$, is close to the microscopic friction, $\bar{\eta} \sim \eta$. There exists a hysteresis behavior for the depinning transition with unequal critical forces $F_{a}$ and $F_{b}$ for increasing and decreasing external force $F_{x}$, corresponding to the static and kinetic friction forces, respectively. On the other hand, in the dense disorder limit $x_{d}=1$ of Fig. 2, the hysteresis is completely washed out leading to equal static and kinetic friction forces $F_{a}=F_{b}$.

Recently Carlin et al. ${ }^{17}$ have studied the depinning transition under an external driving force for $\mathrm{Kr}$ films on gold. They employed the quartz crystal microbalance technique. In this technique, the quartz crystal is driven to resonance by an external rf voltage with the $\mathrm{Kr}$ film adsorbed on the gold electrodes. The driving force on the admolecules is proportional to the oscillation amplitude $A$ of the electrodes and the resultant response of the $\mathrm{Kr}$ film is measured through the "slipping time" $\tau$ which is inversely proportional to the sliding friction $\bar{\eta}$. For a given coverage of the $\mathrm{Kr}$ film, they found a sharp depinning transition when the oscillation amplitude exceeds a critical value. This transition shows the same kind of hysteresis effects as obtained in our weak disorder result shown in Fig. 1. A very interesting result they found is that the depinning transition changes as a function of aging of the system. As shown in Fig. 3(a), the depinning transition is sharp and shows hysteresis behavior after 1 day. (a)

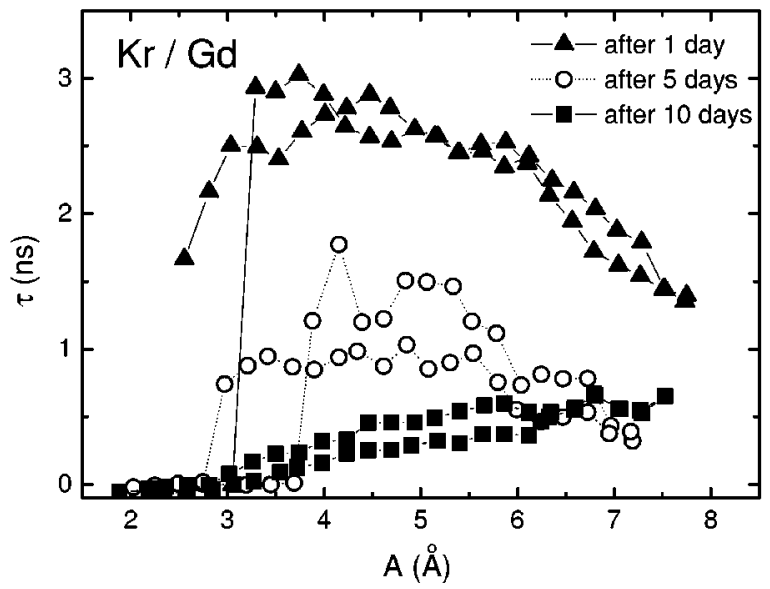

(b)

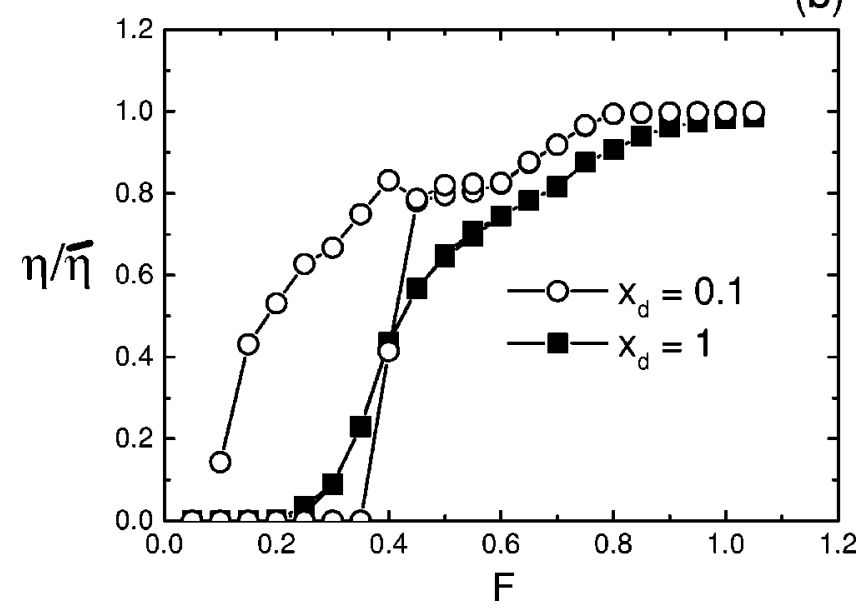

FIG. 3. (a) Slip time $\tau$ as a function of the amplitude of substrate oscillations, obtained from experiments on $\mathrm{Kr} / \mathrm{Gd}$ films using the quartz crystal microbalance technique, by Carlin et al. ${ }^{17}$ (b) Inverse of the nonlinear sliding friction $\bar{\eta}$ (longitudinal), normalized by the microscopic friction parameter $\eta$, as a function of the applied force for weak $\left(x_{d}=0.1\right)$ and strongly disordered $\left(x_{d}=1\right)$ substrate, obtained from the numerical simulations of this work.

However, the corresponding results after 10 days show a rounded transition without hysteresis. The authors speculated that this change is probably due to the deposition of foreign molecules onto the gold electrode substrate, acting then as defect pinning centers. In other words, the disorder of the substrate increases with aging. In Fig. 3(b), we combine the numerical results for $x_{d}=0.1$ and $x_{d}=1$ from the present model to show the effect of increasing density of defect pinning centers on the depinning transition. Our results bear a strong qualitative resemblance to the experimental data shown in Fig. 3(a). In particular, while the weak disorder $\left(x_{d}=0.1\right)$ substrate shows a sharp depinning transition with strong hysteresis effect, the depinning transition for the strong disorder $\left(x_{d}=1\right)$ substrate is smeared out and there is no trace of hysteresis in this case. In fact, our result for the strong disorder case bears a strong qualitative resemblance to the experimental data aged after 10 days. Thus, the theoretical results obtained in our present model study clearly sup- 
port the speculation ${ }^{17}$ that the aging effect on the depinning transition observed in the quartz microbalance experiment is due to the increasing disorder of the substrate. Another interesting feature of the experimental results in Fig. 3(a) is the monotonic decrease of the slip time with aging. It should be noted that this effect is not seen in Fig. 3(b) because the nonlinear sliding friction $\bar{\eta}$ is normalized by the unknown microscopic friction parameter $\eta$, contained in the effective model of Eq. (1). This parameter is essentially the frictional damping of individual adatoms due to coupling to the substrate. As the morphology of the substrate changes, the frictional damping may change significantly. So the monotonic decrease of the slip time in Fig. 3(a) can be accommodated within our model as resulting from an increase in this microscopic frictional damping with aging. The calculation of this microscopic parameter will certainly require a detailed theoretical model for this mechanism including realistic potentials which are outside the scope of the present work.

\section{B. Nature of the sliding state}

To understand the nature of the sliding state at different sliding velocities, it is useful to introduce, just as in the periodic case, ${ }^{3}$ the concept of an effective temperature $T^{*}$ of the sliding overlayer. This is defined via the velocity fluctuation as

$$
T^{*}=m\left(\left\langle\mathbf{v}^{2}\right\rangle-\langle\mathbf{v}\rangle^{2}\right) / 2 .
$$

For a meaningful definition of this effective temperature, one would expect that the velocity distribution should be Gaussian and the width of the distribution related to the effective temperature by Eq. (7). This is confirmed in Fig. 4 where it is shown that the velocity distribution at $F_{x}>F_{c}$ is in fact well described by a Gaussian both for $x_{d}=0.1$ and $x_{d}=1$.

The behavior of the effective temperature $T^{*}$ under the driving force carries important information for the structure properties of the sliding state. As can be seen from Fig. 1, in the dilute disorder case with $x_{d}=0.1$, this effective temperature is much higher than $T$ in the region $F_{b}<F_{x}<F_{c}$ and decreases for $F_{x} \gg F_{c}$, although never approaching $T$ as in the periodic case. This suggests that for weak disorder, the region for $F_{b}<F_{x}<F_{c}$ is strongly disordered and corresponds to a dynamically melted liquid phase. For $F_{x} \gg F_{c}$, the sliding state is a solid state with weak disorder. For the substrate with dense disorder $x_{d}=1$, the effective temperature remains much higher than $T$ even for large driving force, suggesting that in this case the overlayer remains in a liquid like or floating state even at large driving force and high drift velocities. It is interesting to point out that an expression relating $T^{*}-T$ and $\bar{\eta}-\eta$ proposed by Persson ${ }^{3}$ has been found to work well for a periodic pinning potential. According to this relation, in the limit of large driving force $F_{x}$ $>F_{c}, \bar{\eta}$ should approach $\eta$ and $T^{*}$ should approach $T$. Our results in Figs. 1 and 2 show that this expression breaks down in the presence of disorder. While $\bar{\eta}$ does approach $\eta$ in the large driving force limit, $T^{*}$ remains higher than $T$ in this limit in presence of disorder. (a)
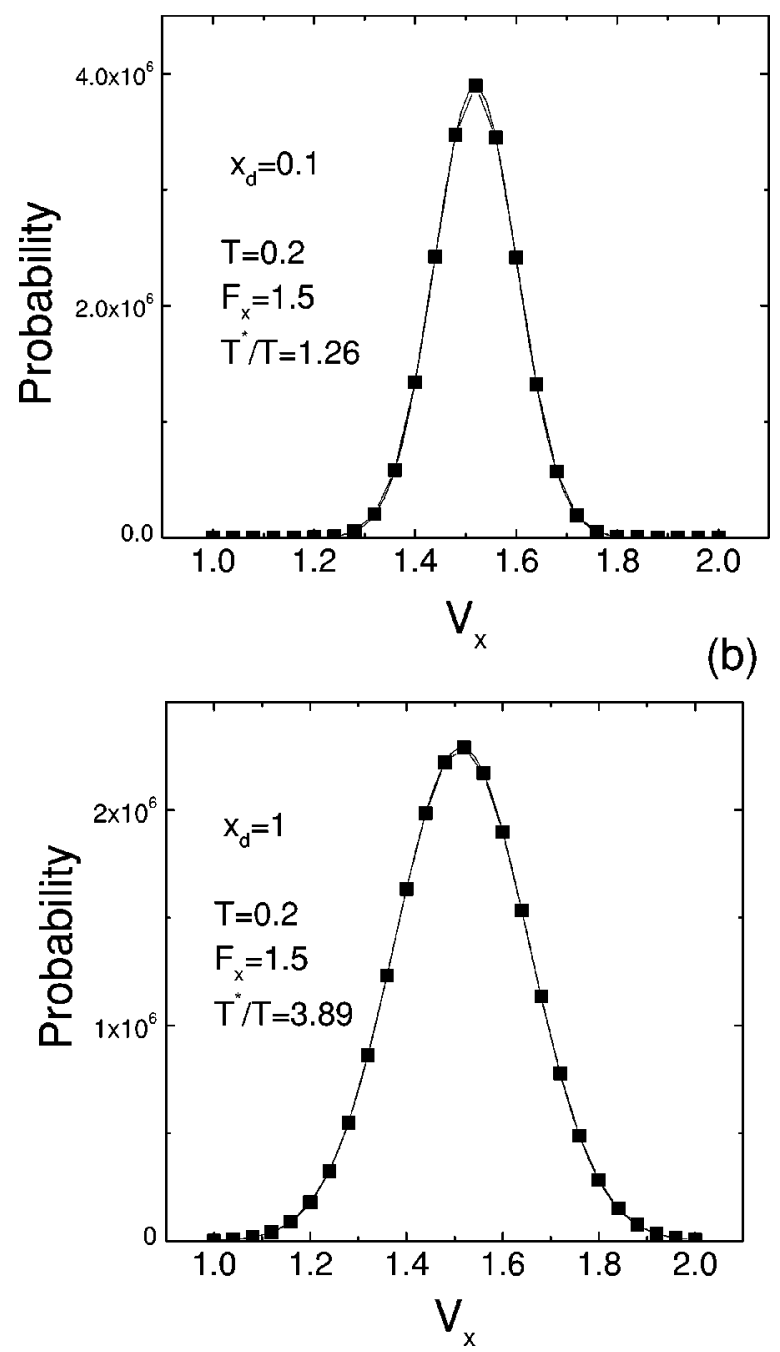

FIG. 4. Velocity distribution at $T=0.2, F_{x}=1.5$, for $x_{d}=0.1$ (a) and $x_{d}=1$ (b). Continuous line is a Gaussian fit.

To support the above interpretation between the effective temperature $T^{*}$ of the sliding overlayer and its structure, we have evaluated the structure factor defined by

$$
S(\mathbf{k})=\frac{1}{N}\left[\left\langle\sum_{m, n} e^{i \mathbf{k} \cdot\left(\mathbf{r}_{m}-\mathbf{r}_{n}\right)}\right\rangle\right]
$$

for large driving force at $F_{x}=1.5, T=0.2$ in both the dilute and dense disorder limit in the present model. The angular brackets denote thermal averages and the square brackets disorder averages. The results are shown in Fig. 5. For a fully ordered configuration the normalized structure factor $S(k) / N=1$ at the peak for a wave vector corresponding to the ordered structure. As expected, for both $x_{d}=0.1$ and $x_{d}$ $=1$ cases, this value is much smaller at the nonzero wave vectors confirming that the sliding layer in the presence of the disorder does not have full long-range order. For $x_{d}$ $=0.1$ the structure factor shows clear prominent peaks at wave vectors corresponding to a commensurate $c(2 \times 2)$ structure, $[\vec{k}=(1,1) \pi / a]$, consistent with a weak disordered 

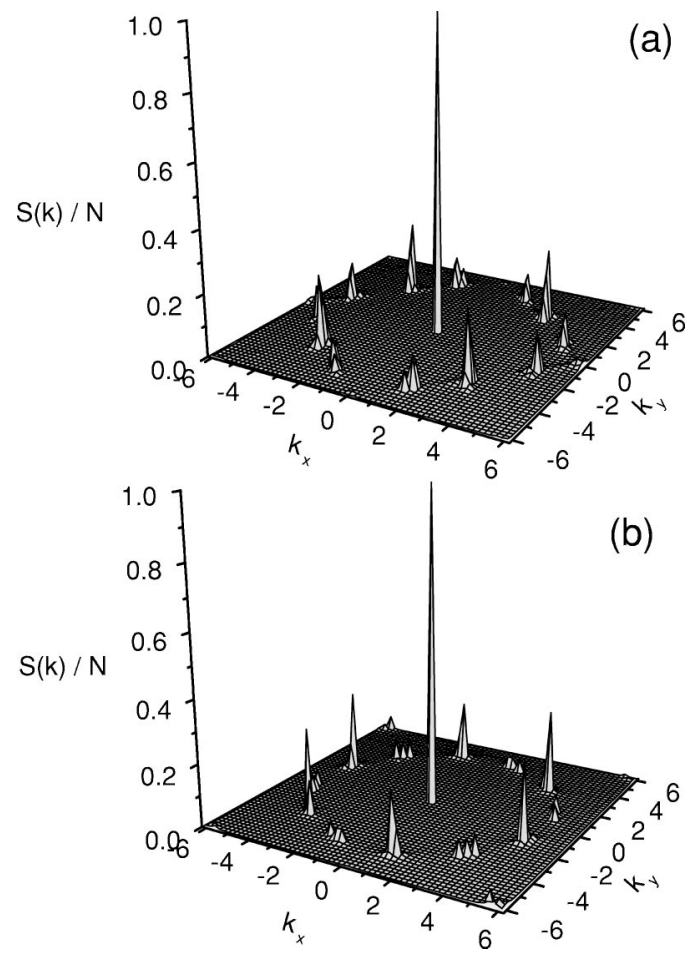

FIG. 5. Structure factor $S(k) / N$ at $F_{x}=1.5, F_{y}=0$ for dilute disorder $x_{d}=0.1$ (a) and dense disorder $x_{d}=1$ (b). The results are for $T=0.2$ and system size $L=30$.

sliding solid state. For $x_{d}=1$, corresponding to the strong disorder limit, one can identify in the structure factor still well defined, although weaker, peaks at the positions corresponding to an incommensurate hexagonal structure. However, the information about the initial periodic substrate no longer exists. The structure of the overlayer in this strong disorder limit can be characterized as an incommensurate floating state both in $x$ and $y$ directions rather than as a liquid state.

Finally, we like to examine how the results presented here depends on the details of the modeling of the disorder and defect pinning centers. In this regard, it is interesting to compare the results presented here with a previous work by Persson $^{3}$ where the disorder was implemented by fixing one of the adsorbate atoms. It was found that in this case, corresponding to $x_{d}=0.0069$ (linear size $L=12$ ), the effective temperature $T^{*}$ increases with increasing force for $F>F_{a}$ and that the sliding incommensurate solid phase observed for the periodic substrate is destroyed and replaced by a fluid state. At first sight this seems to be in total contrast to the results found in this work for the corresponding dilute disorder limit. To understand this difference, we note that in a strict two-dimensional model, all the adsorbate particles are confined to move in the $x-y$ plane only. Hence, the structure of the sliding layer has to undergo strong distortions in the vicinity of the fixed impurity atom, due to the hard-core repulsion of the Lennard-Jones interacting potential, leading to a fluid phase. Thus, in this fixed-impurity model, even a low concentration of impurities actually correspond to strong disorder, in sharp contrast to the model studied here. One would expect that even for the fixed impurity model, a very small

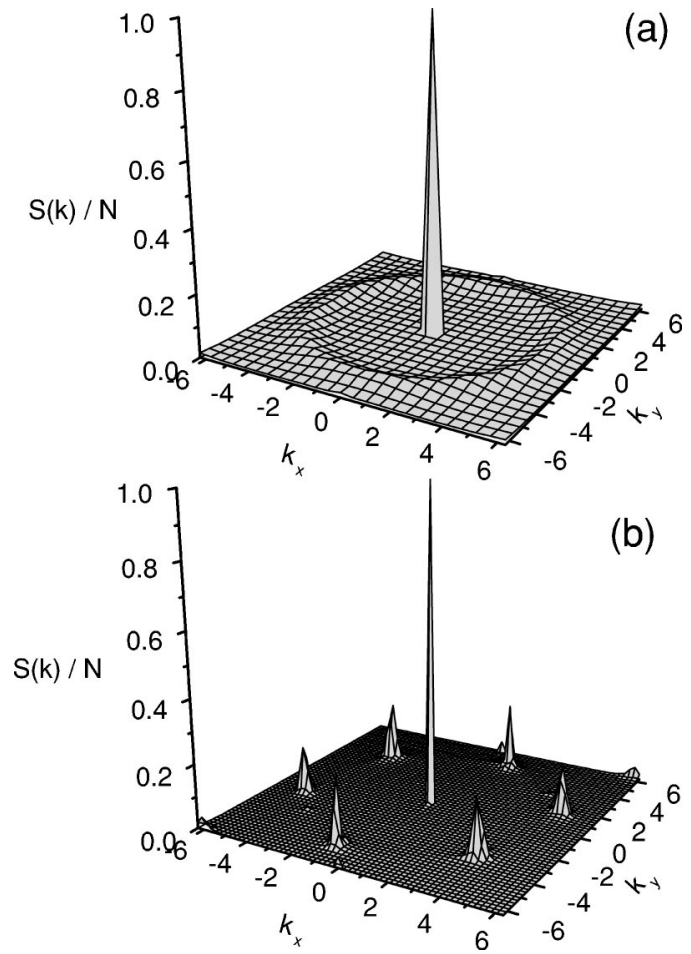

FIG. 6. Structure factor $S(k) / N$ at $T=0.2, F_{x}=1.5, F_{y}=0$ for the periodic substrate and fixing one of the adsorbates at the potential minima for a system size $L=12$ (a) and $L=30$ (b).

concentration of impurities should lead to a more ordered sliding state. To verify this, we have computed the structure factor for the fixed impurity model at two concentration of $x_{d}=0.0069$ ( linear size $L=12$ ) and $x_{d}=0.0012$ (linear size $L=30$ ) in the steady sliding state at $F_{x}=1.5$. For $L=12$ as shown in Fig. 6(a), the corresponding structure factor is characteristic of a fluid phase, in agreement with the findings of Persson. ${ }^{3}$ However, for the larger system size $L=30$ in Fig. 6(b), and therefore more dilute disorder, sharp peaks develop corresponding to an hexagonal structure. This structure is very similar to what we found in the present model for $x_{d}$ $=1$ as shown in Fig. 5(b) indicating that the overlayer in this situation corresponds to an incommensurate hexagonal floating phase.

\section{Transverse response at large driving force}

In this section, we focus our attention on the nature of the sliding state at large driving forces $F_{x}>F_{c}$, and its relation to the response of the sliding layer to an additional transverse driving force. It turns out that the nonlinear response of the overlayer in the transverse direction is sensitive to the nature of the sliding state, thus serving as an ideal probe for the structure of the sliding state which is otherwise difficult to study.

We have studied the transverse response of the overlayer in a steady sliding state along the $x$ direction. For a fixed large driving force $F_{x}=1.5>F_{c}$, an additional variable transverse force $F_{y}$ is applied, and the average transverse drift velocity $V_{y}$ as a function of $F_{y}$ is determined. The resultant transverse response is shown in Figs. 7 and 8. For 
(a)
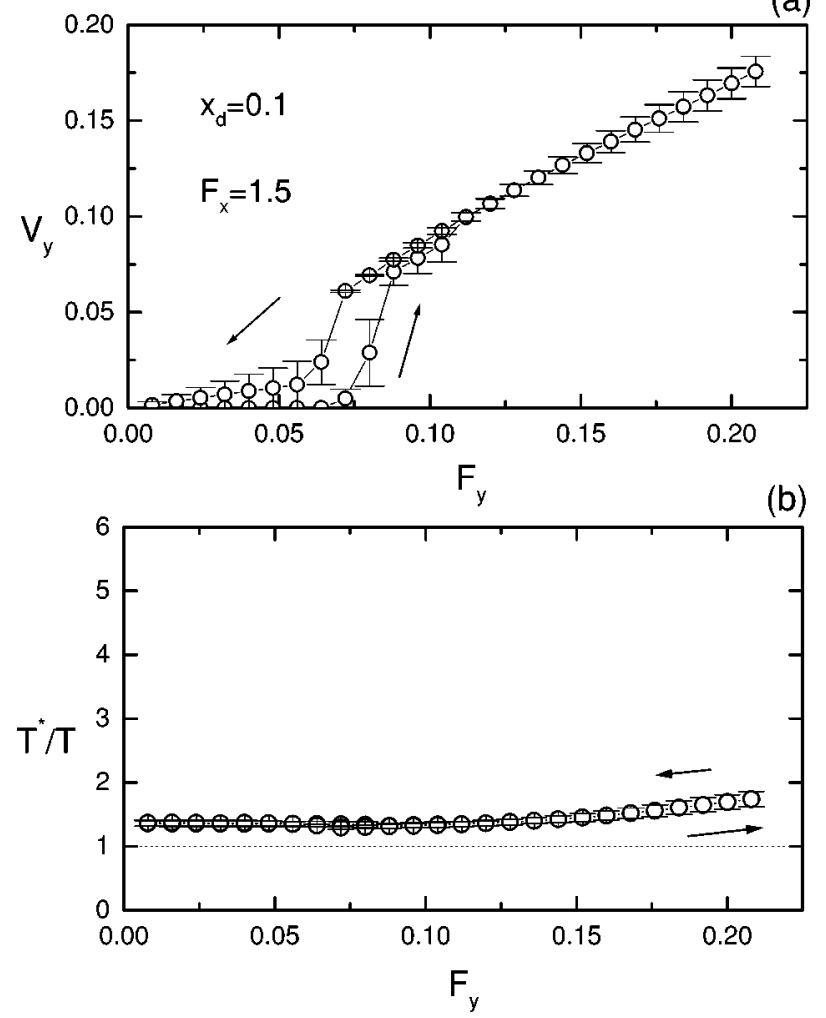

FIG. 7. Transverse velocity $V_{y}$ (a) and effective temperature $T^{*}$ (b) of the overlayer as a function of an additional force along the $y$ axis at fixed $F_{x}$, for dilute disorder $x_{d}=0.1$. Arrows indicate the direction of force variation. Results are for $F_{x}=1.5, T=0.2$, and system size $L=20$.

dilute disorder $x_{d}=0.1$, we find that there is a transverse depinning transition with a critical transverse force $F_{y a}$. This transverse depinning transition is accompanied by hysteresis behavior with the lower critical force $F_{y b} \sim 0$. The existence of this transverse depinning transition for weak disorder is consistent with our finding of the nature of the sliding state at large longitudinal driving force. As shown in Fig. 1, for the sliding state with $F_{y}=0$ and $F_{x}>F_{c}$, we have $T^{*} \sim T$. The structure factor shown for this state in Fig. 5 has prominent peaks at the $c(2 \times 2)$ commensurate structure despite the disorder resulting from a random distribution of the pinning centers. Thus, even while sliding along the $x$ direction, the overlayer can be pinned in the transverse direction by the commensurate pinning potential. This is an origin for the existence of the transverse depinning transition. In the other limit of dense pinning centers $x_{d}=1$ corresponding to strong disorder, the transverse response in Fig. 8 shows no hysteresis and the transverse critical force $F_{y a}$ appears to vanish. Again, this behavior can be understood from the nature of the corresponding sliding state at $F_{y}=0$. As shown in Fig. 2, in this regime of strong disorder, $T^{*} \gg T$ when $F_{x}$ $>F_{c}$. Here the overlayer film is fully disordered and incommensurate with the substrate along both the $x$ and $y$ directions. As a result, there is no pinning force along the transverse direction, hence the absence of a transverse depinning transition.

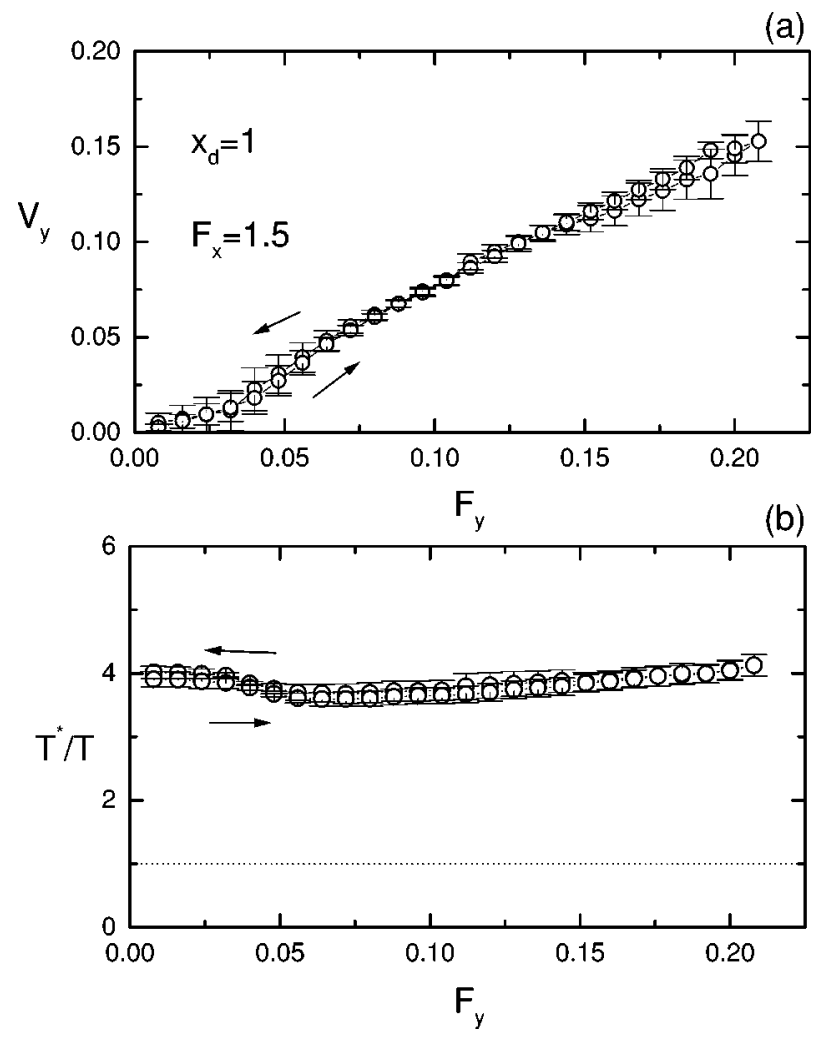

FIG. 8. Transverse velocity $V_{y}$ (a) and effective temperature $T^{*}$ (b) of the overlayer as a function of an additional force along the $y$ axis at fixed $F_{x}$, for dense disorder $x_{d}=1$. Arrows indicate the direction of force variation. Results are for $F_{x}=1.5, T=0.2$, and system size $L=20$.

\section{CONCLUSIONS}

We have studied the nonlinear sliding friction of adsorbed overlayers on disordered substrates with point defects (pinning centers). A simple model is introduced which is a generalization of the driven monolayer model previously used for periodic surfaces. ${ }^{1,3,7}$ The present model incorporates in a convenient way the effects of point defects on an initially ordered substrate. We have studied the longitudinal and transverse response in this model by Brownian moleculardynamics simulations in both the weak and strong disorder limit. The results show that the main features, such as hysteresis, longitudinal, and transverse depinning previously observed for periodic surfaces, ${ }^{3,7}$ still survive in presence of weak disorder but are washed out in the strong disorder limit. The results for longitudinal response bear a strong qualitative resemblance to the recent experimental data of the depinning transition of $\mathrm{Kr}$ films on $\mathrm{Gd}$, using the quartz crystal microbalance technique. ${ }^{17}$ This qualitative agreement supports the interpretation ${ }^{17}$ of the experimental data that the aging effects on the depinning transition are due to the increasing disorder of the substrate. The present model can also be used to gain insight into other interesting features of the experimental results such as the coverage dependence of the depinning transition and sliding friction. We expect that the coverage dependence in the weak disorder limit to be similar to 
that obtained in numerical simulations for a periodic substrate $^{3}$ but quite different in the strong disorder limit. Moreover, within the present model, a study of the nucleation of topological defects and detailed microscopic mechanism of the depinning transition itself should be interesting for driven lattice systems in general.

\section{ACKNOWLEDGMENTS}

This work was supported by a NSF-CNPq international collaboration grant (SCY and E,G,) and Fundação de Amparo à Pesquisa do Estado de São Paulo-FAPESP (E.G.) (Grant No. 03/00541-0).
${ }^{1}$ B.N.J. Persson, Sliding Friction: Physical Principles and Applications (Springer, Heidelberg, 1998).

${ }^{2}$ Physics of Sliding Friction, edited by B.N.J. Persson and E. Tosatti (Kluwer, Dorbrecht, 1996).

${ }^{3}$ B.N.J. Persson, Phys. Rev. Lett. 71, 1212 (1993); Phys. Rev. B 48, 18140 (1993); J. Chem. Phys. 103, 3449 (1995).

${ }^{4}$ P.A. Thompson and M.O. Robbins, Science 250, 792 (1990).

${ }^{5}$ O.M. Braun, T. Dauxois, M.V. Paliy, and M. Peyrard, Phys. Rev. Lett. 78, 1295 (1997).

${ }^{6}$ E. Granato and S.C. Ying, Phys. Rev. B 59, 5154 (1999); E. Granato, M.R. Baldan, and S.C. Ying, in Physics of Sliding Friction, edited by B.N.J. Persson and E. Tosatti (Kluwer, Dorbrecht, 1996), p. 103.

${ }^{7}$ E. Granato and S.C. Ying, Phys. Rev. Lett. 85, 5368 (2000).

${ }^{8}$ M. Das, S. Ramaswamy, and G. Ananthakrishna, Europhys. Lett. 60, 636 (2002).

${ }^{9}$ L. Gao, J. Gu, and B. Hu, Phys. Rev. B 66, 064309 (2002).

${ }^{10}$ A. Vanossi, J. Röder, A.R. Bishop, and V. Bortolani, Phys. Rev. E 63, 017203 (2001)

${ }^{11}$ D.J. Diestler, J. Chem. Phys. 117, 3411 (2002).

${ }^{12}$ T. Giamarchi and P. Le Doussal, Phys. Rev. Lett. 76, 3408 (1996); P. Le Doussal and T. Giamarchi, Phys. Rev. B 57, 11356 (1998).

${ }^{13}$ L. Balents, M.C. Marchetti, and L. Radzihovsky, Phys. Rev. B 57, 7705 (1998).
${ }^{14}$ J.N. Israelachvili, Surf. Sci. Rep. 14, 109 (1992); H. Yoshizawa, P. McGuiggan, and J. Israelachvili, Science 259, 1305 (1993); B. Bhushan, J.M. Israelachvili, and U. Landaman, Nature (London) 374, 607 (1995)

${ }^{15}$ M. Heuberger, C. Drummond, and J. Israelachvili, J. Phys. Chem. B 102, 5038 (1998).

${ }^{16}$ L. Bruschi, A. Carlin, and G. Mistura, Phys. Rev. Lett. 88, 046105 (2002).

${ }^{17}$ A. Carlin, L. Bruschi, M. Ferrari, and G. Mistura, Phys. Rev. B 68, 045420 (2003).

${ }^{18}$ C. Reichhardt and F. Nori, Phys. Rev. Lett. 82, 414 (1999).

${ }^{19}$ E. Granato, T. Ala-Nissila, and S.C. Ying, Phys. Rev. B 62, 11834 (2000).

${ }^{20}$ M. Hilke, S. Reid, R. Gagnon, and Z. Altounian, Phys. Rev. Lett. 91, 127004 (2003).

${ }^{21}$ K. Moon, R.T. Scalettar, and G.T. Zimanyi, Phys. Rev. Lett. 77, 2778 (1996).

${ }^{22}$ C. Reichhardt, C.J. Olson, Phys. Rev. B 65, 100501 (2002).

${ }^{23}$ C.C.D. Silva and G. Carneiro, Physica C 391, 203 (2003).

${ }^{24}$ G. Grüner, Rev. Mod. Phys. 60, 1129 (1988).

${ }^{25}$ A. Pertsinidis and X.S. Ling, Phys. Rev. Lett. 87, 098303 (2001).

${ }^{26}$ M.P. Allen and D.J. Tildesley, Computer Simulation of Liquids (Oxford University Press, New York, 1993). 